\title{
LA PROTECTION AUX ÉTATS-UNIS DES OEUVRES D'ART
}

\section{Jane Ginsburg*}

Les États-Unis sont un marché important d'oeuvres d'art, non seulement pour la vente des tableaux, mais aussi pour l'exploitation de reproductions et d'adaptations des images. Par exemple, en dehors des reproductions traditionnelles telles que celles contenues dans des catalogues et livres d'art et des reproductions sous forme de cartes postales et affiches, une oeuvre d'art originairement conçue comme une expression des beaux arts peut s'exploiter telle par exemple une sortie de bain, du papier peint, voire un décor de poubelle. Dans quelle mesure un artiste peut-il être rémunéré ou même s'opposer à l'exploitation commerciale de son oeuvre aux États-Unis?

La loi sur le droit d'auteur des États-Unis de 1976 octroie aux auteurs les droits exclusifs de reproduction, d'adaptation (droit exclusif d'autoriser la confection d'oeuvres dérivées), et de représentation (17 USC § 106). Les auteurs protégés comprennent les créateurs d'oeuvres "picturales, graphiques et sculpturales". Ces oeuvres recouvrent les oeuvres des beaux arts traditionnels, ainsi que les photographies, mais ne comprennent pas les oeuvres d'art appliqué. Ces dernières ne sont protégées que dans leurs éléments décoratifs, et seulement dans la mesure où ceux-ci peuvent se détacher de l'aspect fonctionnel de l'objet (17 USC § 101). En revanche, l'auteur d'une oeuvre picturale, graphique ou sculpturale est investi du droit exclusif d'autoriser la reproduction de son oeuvre sur ou dans un objet fonctionnel (17 USC § 113).

Ainsi, lorsqu'il s'agit d'oeuvres d'art (à l'exception de l'art appliqué), les exploitations mentionnées ci-dessus appartiennent en principe au monopole de l'artiste. Cependant, l'oeuvre d'art ne pourrait bénéficier du monopole aux États-Unis que si elle est protégée par la loi américaine. En plus, même si l'oeuvre est protégée, pour que l'artiste bénéficie de cette protection, il faut qu'il soit titulaire du droit d'auteur. Or, l'existence et la titularité de cette protection aux États-Unis s'avèrent délicate en matière d'oeuvres d'art. Les difficultés découlent de la loi américaine, et notamment de la loi en vigueur jusqu'à 1978 (date d'entrée en vigueur de la loi actuelle de 1976). 
La protection de l'oeuvre d'art dépendra tout d'abord de la date de la création ou de la publication de l'oeuvre, puisque celles-ci détermineront la loi applicable dans le temps. La loi américaine a été refaite en 1976, puis de nouveau modifiée en 1989 et 1990. Le régime de la loi antérieure, celle de 1909, est sensiblement différent de celui de la loi actuelle. Cependant, c'est la loi de 1909 qui déterminera la protection de toute oeuvre publiée avant 1978. Ainsi, il faut d'abord analyser le régime de 1909, régime assez peu favorable aux auteurs, en raison notamment des règles relatives aux formalités et à la durée de protection (I), pour aborder ensuite les changements effectués en 1976 jusqu'à l'introduction en 1990 d'une loi - de portée très réduite - reconnaissant le droit moral des auteurs de certaines oeuvres des beaux arts (II).

\section{L'ancien régime}

Le régime de la loi de 1909 dressait plusieurs obstacles à la protection efficace aux États-Unis des oeuvres d'art. Les droits de l'artiste pouvaient disparaître faute d'accomplissement de formalités préalables à la protection (B), ou bien, faute de renouvellement du délai de protection (C). Dans les deux cas, l'artiste défaillant perdait les droits sur son oeuvre qui tombait alors au domaine public. Restait un autre piège, celui de la titularité des droits sur l'oeuvre, puisque, selon le droit de certains Etats fédérés, le transfert de l'exemplaire physique unique d'une oeuvre d'art, tel qu'un tableau, entraînait une présomption de cession du droit d'auteur en faveur de l'acheteur de l'oeuvre (A).

\section{A Titularité du droit d'auteur de l'oeuvre d'art}

Bien que la loi américaine prévoie clairement aujourd'hui la distinction entre la propriété de l'exemplaire (par ex le tableau), et la propriété incorporelle du droit d'auteur, en disposant que la vente de l'exemplaire n'entraîne pas en soi la cession du droit d'auteur (17 USC § 202), l'identification du titulaire du droit d'auteur quant aux exemplaires vendus avant 1978 est beaucoup plus hasardeuse. La loi de 1909 prévoyait aussi une distinction entre la propriété de l'objet et celle du droit d'auteur ( $\$ 27$ ), mais cette loi ne s'appliquait qu'aux oeuvres publiées. Si l'oeuvre était inédite, c'était le droit des Etats fédérés qui régissait la titularité du droit d'auteur. Or, les juridictions de certains Etats fédérés, notamment celle de New York, avaient énoncé une présomption de cession du droit avec la vente de l'oeuvre. Il incombait à l'artiste de réserver son droit d'auteur, ou du moins d'apporter quelque limites aux prérogatives de l'acheteur. (Voir, par ex Pushman v New York Graphic Soc 287 NY 203. En 1964, le législateur de New York a adopté une loi instaurant la présomption inverse; cependant, pour les ventes antérieures à 1964, la question de la titularité continue de se poser.) Sans cette réserve de la part de l'artiste, dans plusieurs Etats, la titularité du droit d'auteur se transférait à l'acheteur ensemble avec le tableau. 


\section{B Formalités}

Selon la loi de 1909, l'oeuvre, lors de sa publication, devait porter la mention de réserve; c'est à dire le c. Si l'auteur n'apposait pas la mention, l'oeuvre tombait dans les domaine public. En revanche, tant que l'oeuvre restait non publiée, aucune formalité n'était exigée. La question s'impose alors: en droit américain, qu'est-ce qu'une "publication" d'une oeuvre d'art?

\section{Publication}

La notion générale de publication selon la loi de 1909 était interprétée par les juridictions comme visant la diffusion générale au public des exemplaires de l'oeuvre. $\mathrm{Si}$ cette notion sert à comprendre la publication en matière d'oeuvres littéraires, elle apporte peu à la compréhension de la publication d'oeuvres littéraires, elle apporte peu à la compréhension de la publication d'oeuvres d'art. En effet, qu'en est-il lorsque l'oeuvre n'existe qu'en un exemplaire? Certaines décisions ont retenu la publication même dans l'hypothèse de vente de copie unique, mais les autorités judiciaires sont divisées. Voir en général Academy of Motion Picture Arts and Sciences v Creative House Promotions, Inc $944 \mathrm{~F}$ 2d 1446 (9th Cir 1991) (à propos de la publication de la statuette "Oscar").

Si l'oeuvre est exposée au public, par exemple dans un musée ou dans une galerie, y a-t-il "publication"? Là encore, les autorités judiciaires ne sont pas unanimes, mais certains juges ont statué en faveur d'une publication lorsque l'oeuvre était exposée de telle manière que n'importe quel membre du public pouvait reproduire l'oeuvre. Ainsi, par exemple, un tribunal de district de Chicago a estimé que l'exposition publique d'une maquette d'une sculpture monumentale de Picasso, sans qu'il ne soit interdit au public de prendre des photographies ou de dessiner la maquette, entraînait la publication de l'oeuvre, avec la conséquence que l'oeuvre était jugée tombée dans le domaine public, puisqu'elle était "publiée" sans mention de réserve. Voir Letter Edged in Black Press v City of Chicago $320 \mathrm{~F}$ Supp 1303 (ND I11 1970).

La diffusion de reproductions de l'oeuvre, par exemple dans des livres d'art ou sous forme de cartes postales, équivaut-elle à une "publication"? Là encore, l'unanimité fait défaut. Certains estiment qu'il y a publication de l'image, alors que d'autres rappellent que le livre d'art ou la carte postale ne communique pas l'oeuvre originale, amis une oeuvre dérivée, et que la publication d'une oeuvre dérivée n'entraîne pas la publication de l'oeuvre de base. Sur ce point, l'on pourrait discerner l'existence de la "publication" selon ses conséquences. Lorsque l'auteur souhaitait qu'il y ait publication du tableau par moyen de la publication d'un livre ou d'une autre oeuvre reproduisant le tableau, normalement il apposait la mention de réserve sur le livre ou autre oeuvre. Cette mention permettait de conserver globalement le droit d'auteur sur tout le contenu du livre, et, selon le Copyright Office, la publication était considérée effectuée pour tout le contenu, y compris le tableau. 
En revanche, si l'oubli de l'auteur aurait conduit à la chute de permettait l'application du principe selon lequel les juges ne doivent pas retenir une qualification de "publication" si le résultat était de faire perdre à l'auteur son droit. Voir Academy of Motion Picture Arts $\mathcal{E}$ Sciences, supra. Dans ce cas, un tribunal pouvait décider que la publication sans mention de réserve, par exemple, de cartes postales du tableau n'impliquait pas la perte de protection de l'oeuvre elle-même.

\section{Apposition de la mention de réserve}

Si l'oeuvre était estimée "publiée" avant mars 1989, date d'entrée en vigueur de l'adhésion des États-Unis à la Convention de Berne, l'apposition de la mention s'imposait. En ce qui concerne les publications ayant lieu entre 1978 et mars 1989, l'obligation de la mention s'appliquait non seulement aux oeuvres publiées aux États-Unis, mais aussi à celles publiées partout dans le monde. Ainsi, une Cour d'appel, interprétant la disposition de la loi américaine qui prévoit la mention à partir de la publication "aux États-Unis ou ailleurs" 17 USC § 401(a), a statué que, pour bénéficier du droit d'auteur aux États-Unis, un auteur japonais était tenu d'apposer la mention lors de la première publication au Japon, et cela même lorsqu'aucune mention n'était exigée par la loi Japonaise, et que l'oeuvre n'était pas encore commercialisée aux États-Unis. Voir Hasbro Bradley v Sparkle Toys 780 F 2d 189 (2d Cir 1985).

En ce qui concerne les oeuvres publiées avant 1978, et donc régies par la loi de 1909, les autorités sont divisées quant à la nécessité d'apposer la mention lors d'une première publication en dehors des États-Unis. La Copyright Office soutient que toute oeuvre publiée depuis l'adhésion des États-Unis à la Convention Universelle en 1955 devait porter la mention, quel qu'ait été le lieu de sa première publication. Voir, Compendium of Copyright Office Practices 8.2.1 (1973). En revanche, certaines décisions récentes remettent cette doctrine en cause, en soutenant que l'obligation de la mention ne s'appliquait, selon la loi de 1909 qu'aux exemplaires diffusés aux États-Unis. Voir, Eisen, Durwood \& Co v Tolkein 794 F Supp 85 (SDNY 1992).

\section{Durée de la protection}

Selon la loi de 1909 et les dispositions transitoires de la loi de 1976, le calcul de la durée de la protection dépend en premier lieu du statut de l'oeuvre, à savoir si elle a été publiée ou non.

\section{Oeuvres créés, mais non publiées avant 1978}

Les oeuvres n'ayant pas fait l'objet d'une publication avant 1978 sont protégées durant la vie de l'auteur et jusqu'à cinquante années suivant sa mort. Cependant, même si l'auteur est mort avant 1944, la loi de 1976 prévoit la protection de ses oeuvres inédites jusqu'à 
2002, et, si elles sont publiées entre 1978 et 2002, elles bénéficieront d'encore 25 ans de protection.

\section{Oeuvres publiées avant 1978}

Les oeuvres publiées avec mention de réserve sous le régime de la loi de 1909 bénéficiaient d'une période de 28 ans de protection, renouvelable pour une période supplémentaire de 28 ans. (Une disposition transitoire de la loi de 1976 à ajouté 19 ans à la seconde période de protection, pour faire un totale de 75 ans à compter de la date de la première publication.) Si l'auteur n'inscrivait pas l'oeuvre au Copyright Office et ne demandait pas le renouvellement de l'inscription durant la 28 eme année de protection, l'oeuvre tombait dans le domaine public à l'expiration de 28 ans à compter de la date de première publication - quel qu'ait été le lieu de cette publication. L'on peut noter que l'inscription de l'oeuvre auprès du Copyright Office n'était pas préalable à la protection pendant la première période, mais était nécessaire pour obtenir un second délai de protection, puisque celui-ci dépendait du renouvellement de l'inscription. Cette inscription, néanmoins, pouvait s'effectuer même durant la 28 ieme année de protection.

Ainsi, toute oeuvre publiée avant 1918 est déjà dans le domaine public aux États-Unis, la durée maximale des oeuvres publiées et renouvelées dans le cadre de la loi de 1909 étant de 75 ans. Toute oeuvre publiée entre 1918 et 1963 a dû faire l'objet d'un renouvellement au Copyright Office. A défaut du renouvellement, l'oeuvre est déjà dans le domaine public aux Etats Unis. Pour toute oeuvre publiée entre 1964 et 1978, une loi de 1992 en prévoit le renouvellement automatique. Ainsi, ces oeuvres ne tomberont pas dans le domaine public faute de renouvellement. Les oeuvres publiées à partir de 1978 bénéficient d'une durée unique de la vie de l'auteur plus 50 ans.

\section{La Protection des oeuvres d'art selon la loi actuelle}

\section{A Droit Patrimonial}

Le détenteur du droit d'auteur sur une oeuvre d'art bénéficie du droit exclusif de reproduction, quelle que soit la forme de l'exploitation. Par exemple, la reproduction non autorisée d'une oeuvre sculpturale est une contrefaçon lorsqu'un tiers la reproduit en tant que sculpture, mais également lorsqu'un tiers la reproduit en tant qu'un élément d'un objet fonctionnel, tel qu'un corps de lampe. Ainsi, la loi octroie aux détenteurs du droit d'auteur le contrôle su l'exploitation commerciale, aussi bien que sur l'exploitation "artistique" de l'oeuvre d'art. Mais, si une oeuvre d'art est protégée contre l'exploitation "industrielle" non autorisée, il ne s'ensuit pas que tout objet industriel soit l'objet du droit d'auteur. La loi américaine n'applique pas la règle de "l'unité de art". Au contraire, elle essaie de distinguer entre, d'une part, une oeuvre "picturale, graphique ou sculpturale", faisant l'objet du droit d'auteur, et, d'autre part, un objet fonctionnel dont la forme peut avoir des qualités 
esthétiques, mais qui ne relève que du domaine des dessins et modèles. Comme on la verra, la mise en oeuvre de cette distinction ne s'avère pas totalement heureuse (1).

En plus, même si l'oeuvre rentre dans le domaine du droit d'auteur, ce n'est pas toujours son créateur que la loi réputera "l'auteur" et détenteur du droit d'auteur. Le régime des "works made for hire" peut priver certains artistes, notamment des salariés et, dans certaines hypothèses, des artistes de commande, de la titularité du droit d'auteur, en faveur de l'employeur ou du donneur d'ordre (2).

Enfin, quant à la protée de la protection, il a déjà été noté que l'oeuvre est protégée contre la reproduction non autorisée, y compris par moyen du "merchandising". Cependant, pour que la protection du droit d'auteur joue, il faut que l'objet de la reproduction ait été une oeuvre particulière; normalement, le droit d'auteur ne protège pas contre l'exploitation non autorisée du "style" d'un artiste. Néanmoins, le droit aux "oeuvres dérivées" peut être conçu comme recouvrant des variations visuels sur un thème esquissé par le premier artistepeintre. En plus, une décision récente en matière de concurrence déloyale indique que, dans une certaine mesure, un artiste peut interdire l'exploitation d'une oeuvre tierce qui ressemble aux oeuvres de l'artiste, mais qui n'en est pas en fait une copie (3).

\section{Objet de la protection}

Pour qu'une oeuvre d'art soit recouverte par le droit d'auteur aux États-Unis, il faut qu'elle n'ait pas de caractère fonctionnel. La loi définit une oeuvre fonctionnelle (dénommée "article d'utilité") comme suit:

L'article d'utilité est un article qui remplit une fonction utilitaire intrinsèque ne consistant pas seulement à présenter l'apparence d'un article ou à transmettre des informations. L'article qui fait habituellement partie d'un article d'utilité est considéré comme un "article d'utilité".

Lorsque l'oeuvre possède des caractéristiques à la fois fonctionnelles et artistiques, les éléments artistiques ne seront protégés par le droit d'auteur que s'ils sont "séparables" de l'aspect fonctionnel. La notion de la "séparabilité" en droit d'auteur américain n'est pas facile à saisir, ni pour les juridictions, ni pour la doctrine. Voir en général, Shira Perlmutter Conceptual Separability and Copyright in the Designs of Useful Articles 37 J Copyright Soc'y 339 (1990). Il est certain qu'il s'agit d'une conception plus restrictive que celle de la "multiplicité des formes", selon laquelle la forme d'une oeuvre fonctionnelle serait protégée dès lors qu'il existe un pluralité de formes alternatives qui permettraient à l'oeuvre de fonctionner. Pour être "séparables", il semblerait nécessaire que les éléments artistiques puissent se détacher physiquement de l'objet utilitaire. Bien que la doctrine et certaines décisions essaient de mettre en oeuvre la notion d'une "séparabilité conceptuelle", qui serait plus large que la "séparabilité physique", les autorités n'ont pas réussi à définir cette notion de façon précise, ni de façon qui recueille l'approbation des juges. Il en résulte que plusieurs oeuvres du "design" ne bénéficient pas du droit d'auteur. 


\section{Titulaires du droit d'auteur sur les oeuvres d'art}

Selon le régime américain des "works made for hire", le statut d'auteur et la titularité du droit d'auteur sur une oeuvre créée par un salarié, ainsi que sur certaines oeuvres de commande, reviennent non à l'artiste qui a créé l'oeuvre, mais à son employeur, ou à son donneur d'ordre. La plupart des artistes ainsi déchus de leurs droits travaillent dans les domaines des arts graphiques et de la photographie, et notamment dans les secteurs de la presse et de la publicité. Ces artistes sont soit des salaries, soit des créateurs agissant sur commande.

\section{(a) Oeuvres des salariés}

Mais il faut bien distinguer entre des salariés, et oeuvres de commande. Le droit d'auteur sur une oeuvre de salarié appartient automatiquement à l'employeur, dès lors qu'une relation d'emploi est acquise (même s'il n'y a pas de contrat écrit), et que l'oeuvre a été créée dans l'exercice des fonctions du salarié. Ainsi, les artistes embauchés par des cabinets, notamment par des agences de publicité, ne bénéficient pas du droit d'auteur.

Néanmoins, il est important de contrôler l'existence d'une véritable relation d'emploi. Depuis la décision de la Cour suprême dans Community for Creative Non Violence v Reid 490 US 730 (1989) où la Cour a nié la qualification de "salarié" à un sculpteur qui avait créé une oeuvre selon la commande d'un organisme de bienfaisance pour les sans-abri, les juridictions inférieures refusant de reconnaître la qualité d'employeur à une personne dès lors que cette dernière aura eu le droit d'exiger et de surveiller la création de l'oeuvre. Selon la jurisprudence de la Cour Suprême, il faut la présence de plusieurs éléments avant qu'une relation d'emploi ne soit constituée. Les juges du fond ont retenu qu'une telle relation se caractérise notamment par le paiement par le prétendu employeur des frais pour la sécurité sociale de celui qu'il prétend être son employé. Voir, par exemple, Marco v Acccent Pub 969 F 2d 1547 (3d Cir 1992), rejetant la qualification de "salarié" d'un photographe que travaillait souvent pour une revue, mais qui travaillait à son propre atelier avec ses propres moyens techniques, et dont la revue ne payait jamais de sécurité sociale.

\section{(b) Oeuvres de commande}

Pour qu'une oeuvre de commande soit réputée une "work made for hire", il faut que cette oeuvre rentre dans certaines catégories énumérées de façon limitative dans la loi, et en plus, qu'il y ait un écrit entre le créateur et le donneur d'ordre selon lequel l'oeuvre sera considérée comme une "work made for hire". Les catégories prévues par la loi comprennent les oeuvres collectives, telles que les journaux et les revues. Elles recouvrent aussi les oeuvres audiovisuelles, ainsi que les illustrations accompagnant les oeuvres littéraires. La loi ne précise pas, cependant, ni la forme ni le moment de rédaction de cet écrit. Deux décisions récentes ont interprété la loi en faveur des artistes-créateurs, en statuant que l'écrit doit s'établir au début de la commande. Voir, Schiller \& Schmidt, Inc v Nordisco Corp 
969 F 2d 410 (7th Cir 1992); Playboy Ents v Dumas (SDNY Sept 9, 1993). Dans cette dernière décision, le tribunal a également repoussé l'argument du donneur d'ordre selon lequel l'écrit requis par la loi pouvait se constituer par un cheque sur lequel le donneur d'ordre avait porté la mention que l'encaissement du cheque par l'artiste valait son accord que l'oeuvre serait réputé "work make for hire". Ainsi, par exemple, pour qu'une photographie devienne "work made for hire", il faudrait que les parties se mettent d'accord, avant la création de l'oeuvre, sur sa titularité, et qu'ils consacrent cet accord par un écrit convenable.

\section{Portée de la protection}

L'artiste (ou le détenteur du droit d'auteur) peut interdire l'exploitation non autorisée de son oeuvre par moyen de reproduction classique (par exemple, publication dans un livre, affiches, cartes postales), aussi bien que par le "merchandising", c'est à dire l'incorporation de l'image sur une variété de produits commerciaux, tels que t-shirts, serviettes de bain, peluches, etc. Voir 17 USC § 113. Mais qu'en est-il lorsqu'un tiers exploite non une oeuvre particulière de l'artiste, mais plutôt son style artistique? Prenons deux cas de figure. Dans le premier, supposons que l'artiste a créé une oeuvre dont un tiers reprend certains éléments, par exemple les personnages ou un décor, pour les incorporer dans une oeuvre tierce. Dans le second, supposons la confection d'une image "style Picasso" commercialisée sur des foulards. S'il avait été question de l'apposition sur un foulard d'une reproduction des "demoiselles d'Avignon", la succession Picasso aurait été en droit de s'y opposer. Mais lorsqu'il s'agit d'une image que ressemble à la production artistique de Picasso, mais qui ne correspond pas en fait à aucune de ses oeuvres, le droit d'auteur s'étend-il aussi loin?

Le premier cas s'inspire de la décision du tribunal fédéral de district de New York dans l'affaire Steinberg v Columbia Pictures 663 F Supp 706 (SDNY 1987). En l'occurence, un dessin de New York, reproduisant quelle vision un habitant myope de New York peut avoir de sa ville (à savoir, avec deux rues du côté Ouest de la ville rendues dans tous leurs détails, et le reste du monde en rétréci) avait été repris et puis remanié pour une affiche du film "Moscou à New York" (l'affiche montrait la même perspective visuelle du myope, mais avec deux rues du côté Est de la ville, et le reste du monde en rétréci). Le tribunal a estimé que le défendeur s'était approprié non seulement l'idée d'une perspective myope, mais aussi la façon particulière dont l'artiste Steinberg dessinait cette perspective, le défendeur ayant non seulement copié le style de Steinberg, mais ayant aussi copié certains détails précis de l'oeuvre de Steinberg.

Le second cas s'inspire de la décision dans l'affaire Romm Art v Simbha Int'l 786 F Supp 1126 (EDNY 1992), où il s'agissait de l'imitation par une artiste nommé Patricia du style de l'artiste Tarkay dans sa série d'affiches "Femmes aux cafés". Les deux séries d'affiches montraient des femmes assises dans de différents cafés. L'éditeur de Tarkay avait agi non en contrefaçon du droit d'auteur, mais en concurrence déloyale. Il soutenait que vu la 
proximité des oeuvres de Patricia au style de Tarkay, il y avait un risque de confusion de la part du publique entre les oeuvres des deux artistes, et qu'une oeuvre de "Patricia" pouvait donc être prise pour un "Tarkay". Le tribunal a donné raison à l'éditeur de Tarkay. En effet, le sujet des deux séries d'affiches, ainsi que leur style se ressemblent. Néanmoins, la protection du "style" d'un artiste, que ce soit par le droit d'auteur ou par le droit de la concurrence déloyale, peut être considéré une démarche contestable. D'ailleurs, l'on peut observer que le style de Tarkay ressemble à un Matisse croisé avec un Modigliani. Devraiton pour autant en conclure que Tarkay est redevable des droits d'auteur à ces deux artistes antérieurs?

\section{B La protection du droit moral des auteurs des oeuvres d'art depuis l'adhésion des États-Unis à la Convention de Berne}

Si lors de l'adhésion à la Convention de Berne, les Etats Unis n'ont pas adoptée une protection fédérale explicite du droit moral des auteurs, ils ont, en 1990, rajouté à la loi de 1976 des dispositions spéciales prévoyant les droits d'intégrité et de paternité de certains auteurs des oeuvres des beaux-arts). Suite à plusieurs années de tentatives de consacrer le droit moral des auteurs, le législateur américain a voté une loi instaurant les droits de paternité et d'intégrité des auteurs de certaines oeuvres graphiques, sculptural, et photographiques. Cependant, le domaine de cette loi est extrêmement réduite (1), ainsi que sa portée (2).

\section{Domaine de la protection du droit moral}

Ne sont admises à la protection que certaines oeuvres d'art, à savoir, les oeuvres graphiques et sculpturales en copies uniques ou en série limitée à 200 exemplaires, signés et numérotés. Les photographies sont admises, mais seulement s'il s'agit des images faites dans un but d'exposition, et limitées à 200 exemplaires, signés et numérotés. Le texte de la loi comporte plusieurs exclusions du domaine de la protection, notamment, toute oeuvre de salarié (work made for hire), et toute reproduction de l'oeuvre (en dehors de la série limitée).

\section{Protée de la protection du droit moral}

Le domaine restreint de la loi a des incidences sur sa portée. L'exclusion des reproductions d'oeuvres d'art du champ d'application de la loi a pour conséquence de limiter l'action en violation du droit d'intégrité aux atteintes contre l'exemplaire même. Ainsi, en supposant que Marcel Duchamp avait acquis La Joconde et y avait apposé la moustache (et dans l'hypothèse où le tableau n'était pas dans le domaine public), il y aurait eu méconnaissance de la loi américaine (à supposer aussi sa compétence). Mais, puisque Duchamp avait d'abord établi une copie de la Joconde, et avait peint la moustache sur la copie, il ne peut y avoir violation de la loi américaine sur le droit moral (en revanche, si la Joconde avait bénéficié du "copyright" l'établissement de la copie par Duchamp aurait violé le droit patrimonial sur l'oeuvre). 
Il existe d'autres limitations à la protée de cette loi. D'abord, la loi instaure un régime spécial pour les oeuvres d'art incorporées dans des bâtiments lorsque leurs propriétaires envisagent de détruire ou de modifier le bâtiment. Si les oeuvres sont séparables de l'immeuble, le propriétaire doit notifier à l'artiste de son intention de détruire ou de modifier l'immeuble, afin de permettre à l'artiste de retirer l'objet dans le délai de trois mois, aux frais de l'artiste. Si l'artiste n'enlève pas l'oeuvre dans les trois mois, le propriétaire peut procéder à la destruction ou à la modification de l'immeuble, ensemble avec l'oeuvre. Si l'oeuvre ne peut pas se séparer de l'immeuble, sans être endommagée ou détruite, la loi n'impose pas de limites au droit du propriétaire de l'immeuble pour détruire ou modifier son bien. Cependant, dans cette dernière hypothèse, la loi exige, qu'avant d'installer l'oeuvre dans l'immeuble, l'artiste signe un contrat autorisant l'installation de l'oeuvre et reconnaissant que son retrait ne sera pas possible.

Enfin, la loi permet aux artistes de renoncer à leur droit moral, mais seulement par un écrit, signé, qui précise les circonstances pour lesquelles l'artiste renonce à son droit. Ainsi, en principe, une renonciation globale du droit moral de l'artiste ne sera pas valable. Pour prévenir l'éventualité d'abus, le législateur a demandé au Copyright Office de préparer une étude sur la pratique des renonciations. On peut supposer que si les renonciations deviennent partie intégrante des contrats types en la matière, le législateur reprendra la discussion sur la renonciation.

La durée des droits de paternité et de l'intégrité selon la loi fédérale est la vie de l'auteur, avec un renvoi aux lois des Etats fédérés qui compléteraient la durée en ajoutant les 50 ans après la mort de l'artiste.

\section{The protection of works of art in the United States}

The copyright law of the United States of 1976 grants to authors exclusive rights to the reproduction, adaptation (including the exclusive right to authorise the making of derivative works), and of representation (17 USC para. 106). However, a work of art can only benefit from a monopoly position in the United States if it is protected by American law. Furthermore, even if the work is so protected, before there is any benefit of this protection for the artist, it is necessary also that the artist is the holder of the copyright. The existence of entitlement to this protection in the United States turns out to be a very delicate matter as far as works of art are concerned. The difficulties flow from American positive law particularly that which was enforced until 1978 (the date of the coming into force of the present law of 1976). The protection for a work of art depends first of all on the date of creation of the work and on its publication, since the latter will determine which piece of legislation is applicable. American law was reformed in 1976, then amended in 1989 and 1990 (the amending law of 1990 introduced legislative recognition for the 
principle of the moral rights of authors). The system under the previous law, that of 1909 , is significantly different from that of the present law. However, it is the law of 1909 which will determine the amount of protection given to any work published before 1978 .

The system of the law of 1909 presents several obstacles to the effective protection of works of art in the United States. The rights of the artist can disappear because of failure to accomplish the formalities required for protection, or from a failure to renew within the protected period. In both these cases, the artist affected loses rights in the work which then falls into the public domain. There is also another trap; that is the entitlement to rights in works because according to the law of certain states of the federation, the transfer of the sole physical copy of a work of art, such as a painting, includes a presumption of the transfer of the copyright in favour of the purchaser of the work.

The system instituted by the law of 1976 and its subsequent modifications marks a new stage in the protection of works of art in the United States.

As far as property law is concerned, the holder of the copyright in a work of art has the benefit of the exclusive right of reproduction in whatever form. For example, the unauthorised reproduction of a sculptural work amounts to counterfeiting when a third person reproduces it as a sculpture, and also when a third person reproduces it as an element in a functional object such as a lampstand. Thus the law grants to holders of copyright the control over the commercial exploitation of the property as well as the artistic exploitation of a work of art. But if a work of art is protected against unauthorised industrial exploitation, it does not follow that any industrial object can be the subject of copyright. American law does not apply the so-called rule of "the unity of art". On the contrary, it tries to distinguish between a work which is pictorial, graphic, or sculptural which is the subject of copyright on the one hand, and a functional objects whose form can have aesthetic qualities but which arise only from the field of designs and models on the other hand. The operation of this distinction in practice has not been a particularly happy one.

Though at the time of signing the Bern Convention the United States had not adopted a federal protection which was explicit in respect of the moral right of authors, that was done in 1990 by the addition to the legislation of 1976 of special provisions which dealt with the rights of the integrity and paternity of authors of works in the field of fine arts. After several years of attempts to protect the moral right of authors, the American legislator has introduced a law which affirms the rights of paternity and the integrity of authors of certain graphic, sculptural, and photographic works. However, the range of application of this legislation is extremely reduced. 